M. Sivan

S. Glyn-Jones

J. Phillips Hughes

K. Willett

\section{Arterial injury following a low energy pubic rami fracture}

vessels. As far as we are aware, angiography-proven bleeding from branches of the internal and external iliac arteries in a low-energy, stable pubic rami fracture has not been reported before. This case emphasizes the need for admission and careful hemodynamic monitoring in such cases.

Key words Pubic rami fracture • Loco energy $•$ Arterial injury • External iliac • Internal iliac

\section{Introduction}

Pelvic fractures are usually high-energy injuries indicative of significant trauma. Hypotension and significant blood loss are common with skeletally unstable pelvic fractures. Potential sites of intrapelvic bleeding include fractured surfaces and venous or arterial injuries.

Stable pelvic fractures of the pubic rami are uncommonly associated with arterial bleeding. Previously reported cases have involved high-energy shearing forces with branches of internal iliac artery territory being at risk. We report the case of an elderly person in whom a low-energy fall caused a stable pubic rami fracture associated with angiography-proven major hemorrhage from branches of both the internal and external iliac arteries.

\section{Case report}

A 70-year-old woman with moderately severe Alzheimer's disease tripped and fell at home and was unable to get up. She was taken to the emergency department where she was initially hemodynamically stable (blood pressure $100 / 50 \mathrm{mmHg}$; pulse rate, $88 \mathrm{bpm}$ ), with a tender lower abdomen. An anteroposterior radiograph of the pelvis revealed bilateral inferior and left superior pubic rami fractures (Fig. 1). A chest radiograph was normal. There were no other relevant co-morbidities or drug history (anticoagulants).

Within one hour of arrival, she became hypotensive (blood pressure, 50/25 $\mathrm{mmHg}$ ), tachycardic (pulse, 115 bpm) and tachypneic (respiratory rate, $25 \mathrm{~min}^{-1}$ ) and had 


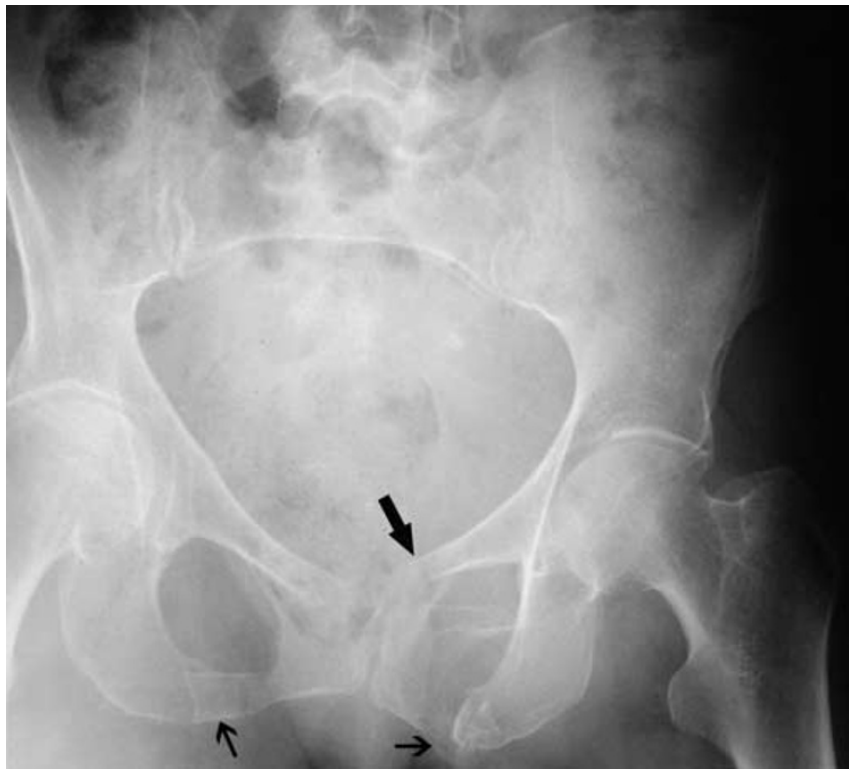

Fig. 1 Plain film demonstrating fractures of both inferior pubic rami (small arrows) and the left superior pubic ramus (large arrow)

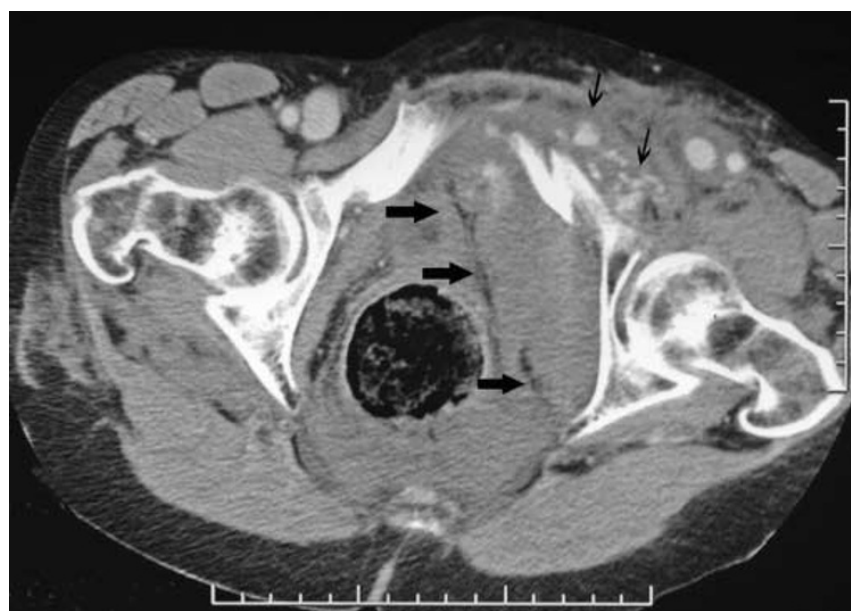

Fig. $2 \mathrm{CT}$ at the level of hip joints demonstrating fresh arterial bleeding (small arrows) and large hematoma (large arrows)

an altered sensorium. On examination, her lower abdomen was distended and tender. Laboratory studies revealed a hemoglobin concentration of $11.8 \mathrm{gm} / \mathrm{dL}$, with normal electrolytes and clotting. She was resuscitated with $2 \mathrm{~L}$ crystalloid and $1 \mathrm{~L}$ colloid and her vital signs improved (blood pressure, 110/50 mmHg; pulse, 94 bpm). Emergency contrast-enhanced computed tomography (CT) of the abdomen and pelvis showed a large pelvic hematoma (Fig. 2). No posterior pelvic ring fracture was identified. Pelvic arteriography demonstrated active arterial bleed from multiple sites in branches of both the anterior and posterior divisions of the internal iliac artery (Fig. 3). The multiple bleeding sites necessitated embolization of the main

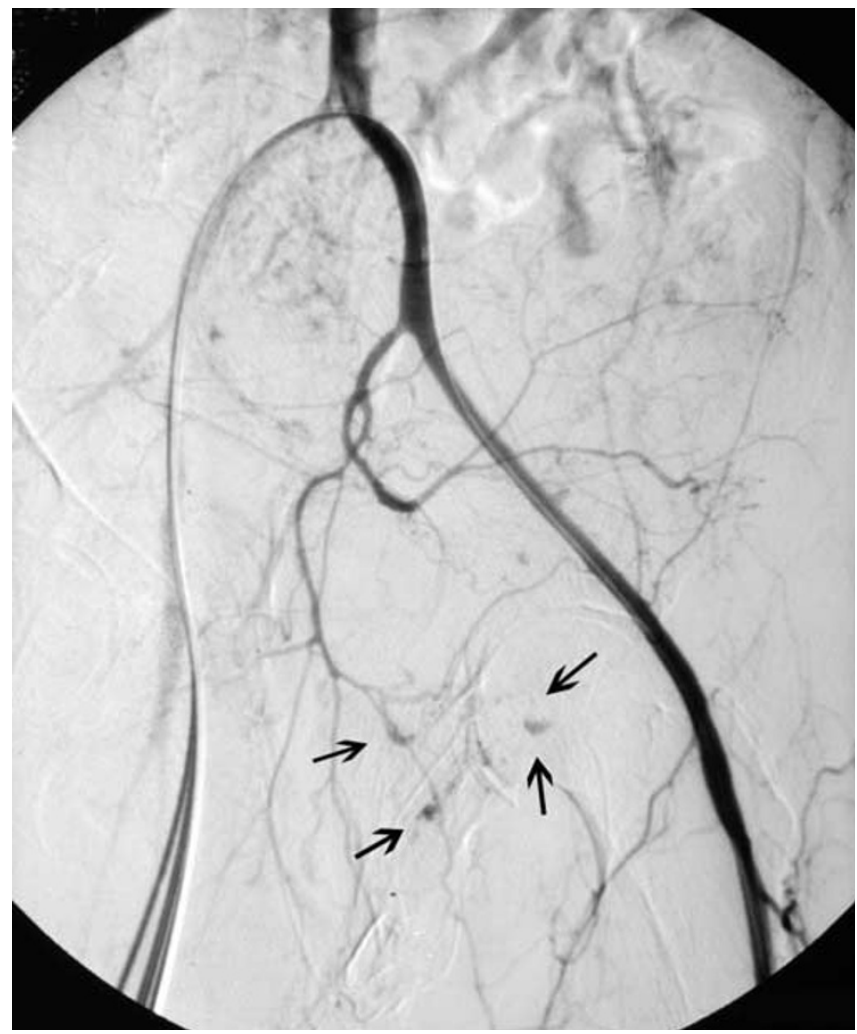

Fig. 3 Left iliac angiogram demonstrating multiple sites of bleeding from the internal iliac branches (arrows)

trunk of the left internal iliac artery with 5-mm coils. There was also bleeding from a branch of the deep circumflex iliac artery (a branch of external iliac artery) on the left side, which was too small and tortuous to allow selective embolization.

The patient remained stable after the intervention. She remained hemodynamically stable but developed a chest infection and respiratory compromise. A chest radiograph showed a large right pleural effusion, which was drained; antibiotics were commenced. Her conditions deteriorated and she died 6 days after injury.

\section{Discussion}

Simple pubic rami fractures without disruption of the posterior pelvic structures are classified as APC I (Young and Burgess classification) [1], or type A (Tile's or OTA classification) [2, 3]. Patients are divided epidemiologically into two main groups: young people who had had highenergy trauma and elderly people who had sustained osteoporotic fractures after minor falls [4]. Cases associated with life-threatening arterial injury mostly relate to the high-energy group [4, 5-8]. Major pelvic hemorrhage 
in the low-energy group is uncommon and rare. Coupe et al. [9] reported a case of fatal hemorrhage following a low-energy pubic rami fracture and hypothesized possible intrapelvic arterial injury, as angiography could not be done on the patient [9]. Our patient had CT-proven stable pubic rami fractures and angiogram-proven arterial bleeding from multiple sites in the internal and external iliac arteries.

Studies to identify arterial bleeding sites with specific fracture classifications have shown that avulsion of the posterior branches of the internal iliac artery is more frequent in unstable posterior pelvic disruptions and bleeding from anterior branches of internal iliac artery in pubic rami fractures $[7,8]$. This patient had bleeding from mul- tiple sites, including a branch of the deep circumflex iliac artery. Interestingly, as far as we are aware, bleeding from both internal and external iliac artery branches subsequent to low-energy pubic rami fractures has not been previously reported. This pattern of bleeding in pubic rami fractures may be explained by the decreased compliance of the vessels to shear forces in this population.

By reporting this case, we wish to emphasize the potential for arterial injury even in apparently stable pubic rami fractures due to low-energy falls in the frail elderly. These patients need admission and careful hemodynamic monitoring. The compromised patients should receive aggressive fluid resuscitation, prompt angiography and embolization of bleeding vessels if indicated.

\section{References}

1. Young JWR, Burgess AR (1987) Radiographic management of pelvic ring fractures: systematic radiographic diagnosis. Urban \& Schwarzenberg, Baltimore

2. Tile M (1996) Acute pelvic fractures: II Principles of management. J Am Acad Orthop Surg. 4:152-161

3. Orthopaedic Trauma Association Committee for Coding and Classification (1996) Fracture and dislocation compendium. J Orthop Trauma 10[Suppl 1]:66-70
4. Perez MUH, Alcover HA (2004) Hypovolaemic shock due to fracture of the superior pubic ramus in a young man. Injury 35:80-82

5. Grainger MF, Porter KM (2003) Life threatening haemorrhage from obturator vessel tear as a result of pubic ramus fracture. Injury 34:543-544

6. Margolies MN, Ring EJ, Waltman AG, Kerr WS, Baum S (1972) Arteriography in the management of haemorrhage from pelvic fractures. N Engl J Med 287:317-321
7. Metz CM, Hak DJ, Goulet JA, Williams D (2004) Pelvic fracture patterns and their corresponding angiographic sources of haemorrhage. Orthop Clin N Am 35:431-437

8. O'Neill P, Riina J, Sclafani S, Tornetta P (1996) Angiographic findings in pelvic fractures. Clin Orthop Relat Res 329:60-67

9. Coupe NJ, Patel SN, McVerry S, WynnJones CH (2005) Fatal haemorrhage following a low-energy fracture of the pubic ramus. J Bone Joint Surg $\mathrm{Br}$ 87:1275-1276 